\title{
Differential Fluorescent Chromosome Banding of Four Species in Haworthia Duval (Aloaceae)
}

\author{
Nasrin Sultana Jessy, Rokeya Begum, Masuda Khatun \\ and Sheikh Shamimul Alam*
}

Department of Botany, University of Dhaka, Dhaka-1000, Bangladesh

Received September 26, 2005; accepted October 24, 2005

\begin{abstract}
Summary The karyotypes of 4 Haworthia species viz. H. papillosa Haw., $H$. fasciata Haw., $H$. limifolia Marloth and $H$. reinwardtii Haw. were compared after differential staining with orcein, CMA and DAPI. In H. papillosa and H. fasciata $2 n=14$ chromosomes were found. H. limifolia and $H$. reinwardtii possessed $2 n=21$ and $2 n=35$ chromosomes, respectively. These 4 species possessed distinct bimodal karyotypes. Two satellites were found one in each on the long arm of 2 large chromosomes of these 4 species. The staining property of satellites was different in each species. In $H$. papillosa, satellites were found in orcein and CMA-staining, negative satellites were found in DAPIstaining. H. fasciata showed satellites in CMA and DAPI, whereas no satellite was found in orcein staining. Two CMA-positive and one DAPI-negative satellites were found in H. limifolia, but none in orcein staining. In H. reinwardtii, satellites were found in orcein staining and not in CMA- and DAPI-staining. No DAPI-positive or negative band was found on the chromosomes in these 4 species. H. papillosa possessed an interstitial stable CMA-positive band on the long arm of a chromosome in pair I. This kind of banded chromosome was absent in other 3 species and thus can be used as a marker. The CMA-banded karyotype of H. limifolia showed a probable occurrence of paracentric inversion and deletion. It also indicated the presence of different genomes with partial homology. Therefore, $H$. limifolia seems to be a segmental allotriploid. $2 n$ chromosome number together with orcein, CMA and DAPI karyotypes suggests $H$. reinwardtii as an autopentaploid.
\end{abstract}

Key words Fluorescent banding, Karyotype, Haworthia, Aloaceae.

The genus Haworthia Duval consists of small succulent plants. Formerly this genus belonged to the tribe Aloineae of the family Liliaceae. Now this tribe is recognized as a family Aloaceae (Brummitt 1992). Haworthia is native and more or less restricted to South Africa. The highest concentration of Haworthia species (spp.) is in the Eastern and Southern Cape regions. This genus has about 68 spp. together with 41 sub-species, varieties and forms (Bose et al. 1991). The members of Aloaceae possessing beautiful flowers with medicinal values. These attracted the cytogeneticists due to their generally large chromosomes (Taylor 1925, Sharma and Chatterjii 1958, Vladimir 1963). In the early seventies, some workers such as Brandham (1969, 1973, 1974, 1975) studied their meiotic and also mitotic chromosomes. From these reports it is now known that the similarity of the orcein stained karyotypes among the members of 3 different genera in Aloaceae viz. Aloe, Gasteria, Haworthia is a noteworthy feature (Darlington and Kefallinou 1957, Vosa and Bayer 1986). Members of these genera possess $2 n=14(x=7)$ chromosomes (8 large and 6 small) with a distinct bimodal karyotype (Brandham 1971). Although the basic karyotype is uniform throughout the family, major structural changes to the chromosomes are widespread with Robertsonian fusions, large translocations and other types of interchanges reported to be frequent. Therefore, species diversification in this family seems to have proceeded without bringing about any change in the mor-

\footnotetext{
* Corresponding author, e-mail: ssalam81@yahoo.com
} 
phology of the karyotypes (Brandham 1969, 1974, Riley and Majumder 1979).

Previous cytogenetical reports of this family were mainly based on analysing their orceinstained karyotypes. Sometimes orcein staining alone is not good enough for analyzing karyotypes, since orcein does not have any base specificity. To overcome this difficulty, we have tried to use the fluorescent chromosome banding technique. The fluorochromes we used here were CMA (Chromomycin $A_{3}$ ) and DAPI (4-6' Diamidino-2-Phenyl Indole). It has been using for critical comparative karyotype analysis (Schweizer 1976, Kondo and Hizume 1982, Hizume et al. 1988, Alam and Kondo 1995, Alam et al. 1995). Since CMA binds to the GC-rich (Guanine-Cytosine) and DAPI to the AT-rich (Adenine-Thiamine) segments of chromosomes, fluorescent banding permits a more detailed and critical chromosome analysis. One can now easily identify the individual chromosome by the appearance of the CMA- and DAPI-bands (positive and negative).

The aim of this investigation was: i) to find out the karyotypic diversification among 4 species after staining with orcein, CMA and DAPI, ii) to determine the distribution and amount of GC- and AT-rich repeates, iii) to elucidate the nature of satellites and iv) to find out the ploidy level of $H$. limifolia and $H$. reinwardtii.

\section{Materials and methods}

The following Haworthia Duval species were used in this study: (i) H. papillosa Haw. (ii) $H$. fasciata Haw. (iii) H. limifolia Marloth. (iv) H. reinwardtii Haw. The materials were collected from the National Botanic Garden, Mirpur, Dhaka-1216, Bangladesh and maintained in the Botanic Garden, Department of Botany, University of Dhaka, Bangladesh.

Roots of all species were collected at about 11.30-12.00 a.m. during December to February while 9.00-9.30 a.m. during March to May. The young healthy roots were cut $c a .0 .5 \mathrm{~cm}$ away from the tip by a clean blade. The collected root tips (RTs) were pretreated with 8-Hydroxyquinoline for $3 \mathrm{~h}$ at room temperature $\left(28-30^{\circ} \mathrm{C}\right)$, followed by $15 \mathrm{~min}$ fixation in $45 \%$ acetic acid at $4{ }^{\circ} \mathrm{C}$. The pretreated RTs were hydrolysed for $3 \mathrm{sec}$ for $H$. papillosa, H. fasciata, H. limifolia and $2 \mathrm{sec}$ for $H$. reinwardtii at $60^{\circ} \mathrm{C}$ in a mixture of $1 \mathrm{~N} \mathrm{HCl}$ and $45 \%$ acetic acid $(2: 1)$ and squashed in $1 \%$ acetoorcein.

For CMA- and DAPI-staining, methods proposed by Alam and Kondo (1995) were followed with slight modifications. After hydrolyzing and dissecting, the materials were tapped and squashed with $45 \%$ acetic acid. The cover glasses were removed quickly and air dried for at least $48 \mathrm{~h}$ before study. The air dried slides were first preincubated in McIlvaine's buffer ( $\mathrm{pH}$ 7.0) for $30 \mathrm{~min}$ followed by distamycin A $(0.1 \mathrm{mg} / \mathrm{ml})$ treatment for $10 \mathrm{~min}$. The slides were rinsed mildly in McIlvaine's buffer supplemented with $\mathrm{MgSO}_{4}(5 \mathrm{mM})$ for $15 \mathrm{~min}$. Then one drop of CMA $(0.1 \mathrm{mg} / \mathrm{ml})$ was added to the materials for $20 \mathrm{~min}$. The slides were treated again for $10 \mathrm{~min}$ in both McIlvaine's buffer with $\mathrm{Mg}^{+2}$ and McIlvaine's buffer without $\mathrm{Mg}^{+2}$. Slides were mounted in $50 \%$ glycerol and kept at $4{ }^{\circ} \mathrm{C}$ for over night before observation. These were observed under a Nikon (UFX-IIA) fluorescent microscope with blue violet (BV) filter cassette. In some cases, the CMA-stained slides were counter stained with DAPI. The slides were rinsed with distilled water for $5 \mathrm{~min}$ followed by destaining in $45 \%$ acetic acid for $15 \mathrm{~min}$. The slides were then washed in distilled water and air dried over night. After destaining, the preparations were immersed in McIlvaine's buffer ( $\mathrm{pH}$ 7.0) for $20 \mathrm{~min}$. The slides were treated in actinomycin $\mathrm{D}(0.25 \mathrm{mg} / \mathrm{ml})$ for $10 \mathrm{~min}$ in a humid chamber. The slides were immersed in McIlvaine's buffer $(\mathrm{pH} 7.0)$ for $10 \mathrm{~min}$ followed by treating in DAPI solution $(0.1 \mathrm{mg} / \mathrm{ml})$ for $15 \mathrm{~min}$ and mounted with $50 \%$ glycerol and kept at least $1 \mathrm{~h}$ at $4^{\circ} \mathrm{C}$. These were observed under a Nikon (UFX-IIA) fluorescent microscope with ultra violet (UV) filter cassette. 
Results and discussion

\section{Orcein stained karyotypes}

H. papillosa was found to possess $2 n=14$ chromosomes (Fig. 1). This species showed heteromorphicity in respect of centromeric position in pair III and IV (Fig. 10). In each pair one chromosome was sub-telocentric and other was acrocentric. This data may indicate a small deletion at the short arm of the respective chromosomes. Gradual decrease in chromosome length was observed in $H$. papillosa, $H$. limifolia and $H$. reinwardtii indicating an asymmetric karyotype in these three species. The karyotype of $H$. fasciata composed of homomorphic pairs showing symmetric karyotypes and thus might be regarded as primitive in nature among the four species studied (Stebbins 1971).

\section{Satellites}

The satellites of four species were unique in nature. In H. papillosa, a pair of satellites was observed at the long arm of chromosome pair III in orcein staining (Fig. 10, arrow). These two satellites stained brightly in CMA (Fig. 2, arrow). No satellite was observed in DAPI stained metaphase chromosomes indicating DAPI-negative feature of the satellites (Fig. 3, arrow). CMA-positive and DAPI-negative properties of satellites in $H$. papillosa reveals that these portions were composed of GC-rich repeats.

No satellite was found in orcein stained metaphase chromosomes of $H$. fasciata (Fig. 4). However, a pair of satellites was observed at the long arm of pair II in both CMA and DAPI-staining (Figs. 5, 6, arrow). The staining of satellites in both CMA and DAPI suggests the existence of GCand AT-rich repeats together. The reason for the absence of satellite in orcein stain is unknown. The chromatin in satellited portions might not be stained with orcein.

In H. limifolia, no satellite was found in orcein staining (Fig. 7). In CMA-staining one satellite was observed on the long arm of a member of pair I (Figs. 8, 12, big arrow) and a member of pair II (Figs. 8, 12, arrowhead). The satellite in pair I was also fluoresced well in DAPI (Fig. 9, big arrow), indicating the presence of GC- and AT-rich repeats together. On the other hand, the satellite in pair II showed negative banding with DAPI (Fig. 9, arrowhead). The reversible staining (CMA-positive and DAPI-negative) indicates GC-rich nature on this satellite portion (Schweizer 1976, Alam and Kondo 1995, Alam et al. 1995, Hizume et al. 1988). Reason for absence of satellite in orcein staining may be same as mentioned above.

Two satellites were observed in orcein stained metaphase of $H$. reinwardtii. These were present on the long arm of two chromosomes in group I out of five chromosomes (data not shown). However, no satellite was detected in CMA- and DAPI-staining (Figs. 11, 13). This portion may consist of some kind of chromatins or DNA that is unable to bind with CMA and DAPI.

Whatever the nature of the satellite, the four species showed a common characteristic. The satellites were present on the long arm of the respective large chromosomes in these four species.

\section{CMA-marker chromosome}

One CMA-positive band was found in H. papillosa. It was located at the interstitial region of the long arm of a chromosome in pair I (Fig. 2, arrowhead). This kind of banded chromosome was not found in the other three species. Therefore, this chromosome can be used as a marker.

\section{H. limifolia}

H. limifolia possessed $2 n=21$ chromosomes (Fig. 7). These 21 chromosomes could be assembled into four large and three small groups. Each group had three chromosomes. Thus the species showed a distinct triploid bimodal karyotype (Fig. 12). The CMA karyotype showed three prominent CMA bands in group V. The location and size of the bands are different. In second and third 


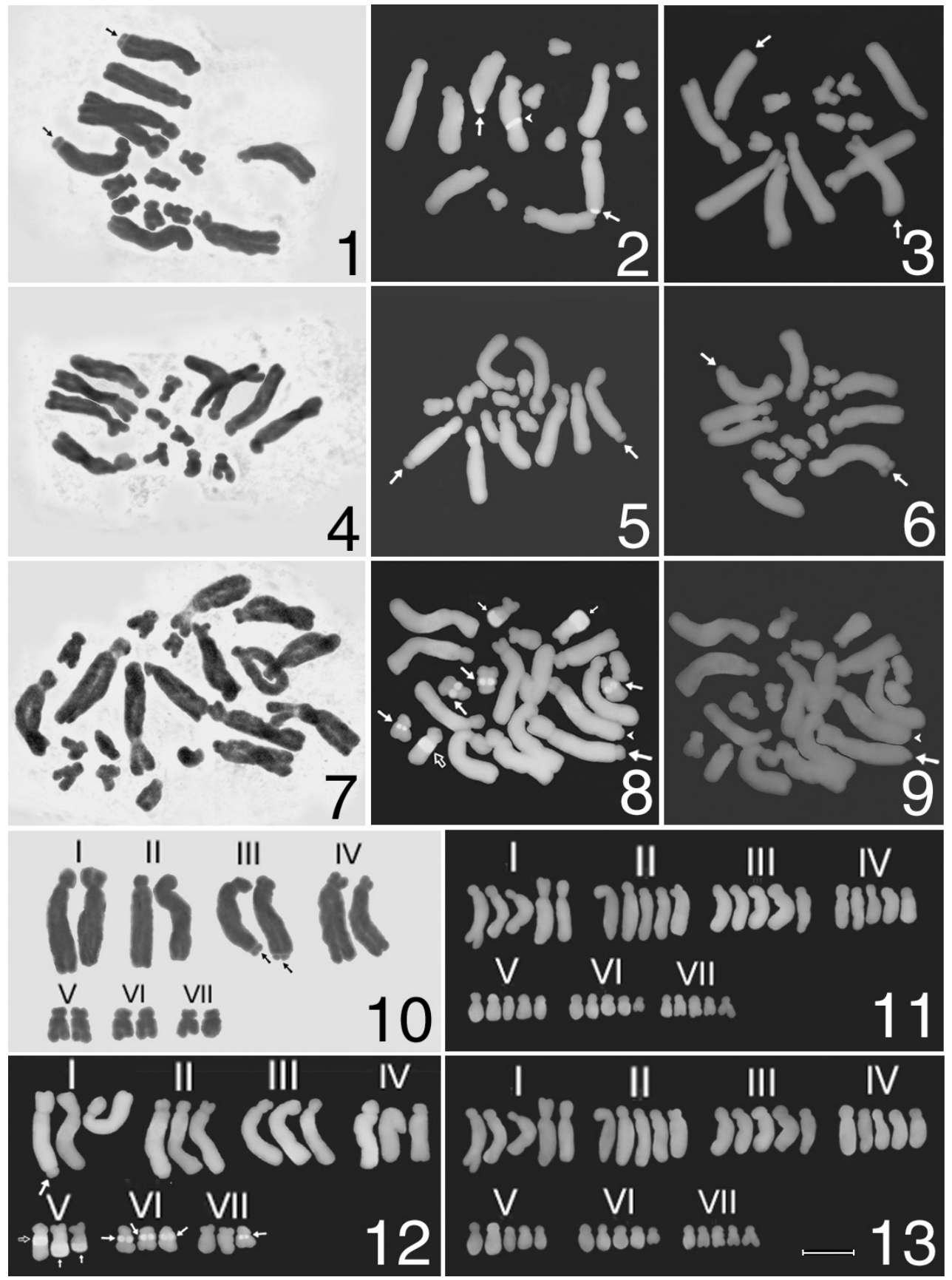

Figs. 1-13. Differential staining of 4 Haworthia spp. 1) Orcein stained metaphase chromosomes of $H$. papillosa, 2) CMA-stained metaphase chromosomes of $H$. papillosa, 3) DAPI-stained metaphase chromosomes of $H$. papillosa, 4) Orcein stained metaphase chromosomes of $H$. fasciata, 5) CMA-stained metaphase chromosomes of $H$. fasciata, 6) DAPI-stained metaphase chromosomes of $H$. fasciata, 7) Orcein stained metaphase chromosomes of $H$. limifolia, 8) CMAstained metaphase chromosomes of $H$. limifolia, 9$)$ DAPI-stained metaphase chromosomes of $H$. limifolia, 10) Orcein stained karyotype of $H$. papillosa, 11) CMA-stained karyotype of $H$. reinwardtii, 12) CMA-stained karyotype of $H$. limifolia, 13) DAPI-stained karyotype of $H$. reinwardtii. Bar $=10 \mu \mathrm{m}$ 
chromosome of this group, CMA-bands were at the terminal end of the long arm (Figs. 8, 12, thin arrow). The CMA-band of third chromosome was much smaller than that of second chromosome. Moreover, the long arm of third chromosome is shorter than that of second chromosome. These features may indicate a deletion of a part from the long arm of third chromosome. If it is considered a deletion in this chromosome, these two chromosomes may be homologous on the basis of their structure and CMA-band. On the other hand, the first chromosome of this group (group V) had a band at the interstitial region of the long arm (Figs. 8, 12, black arrow). The size of this band is almost equal to the band on second chromosome in this group. It indicates a probable occurrence of paracentric inversion on that portion. This is why this chromosome is different from the other two of this group.

In pair VII, only a CMA-positive band was present at the interstitial region of the long arm in a chromosome (Figs. 8, 12, arrow). The other two chromosomes of this group did not show such band. Moreover, the banded chromosome was sub-metacentric whereas the other two non-banded chromosomes of this group were sub-telocentric. This fact suggests the presence of different genomes in this triploid.

Three CMA-positive bands were found one in each member of group VI (Figs. 8, 12, arrow). Their location, size $(1.7 \mu \mathrm{m})$ and intensity were same. The three chromosomes were sub-telocentric and almost same in size. It indicates the complete homology among the three chromosomes of this group.

The CMA-banding property of group V and VII indicates the presence of different genomes. Whereas, group VI showed complete homology. Thus $H$. limifolia seems to possess different genomes with partial homology and may be considered as segmental allotriploid.

\section{H. reinwardtii}

In $H$. reinwardtii, $2 n=35$ chromosomes were observed. These 35 chromosomes could be assembled into four large and three small chromosome sets having five chromosomes in each. Therefore, it showed a distinct pentaploid bimodal karyotype (Figs. 11, 13). No heteromorphicity in respect of centromeric position and length was found. CMA- and DAPI-positive or negative band was absent in this species. The members of each group showed equal staining with CMA and DAPI. Therefore, the overall karyotype suggests this species as autopentaploid.

\section{Karyotype diversification}

The members of these three genera viz. Aloe, Gasteria and Haworthia in Aloaceae possess a similar karyotype of $x=7$ with four large and three small chromosomes (Brandham 1971), which according to him indicates the involvement of minute structural changes in the karyotypic diversification of these genera. Alam and Khanam (2005) reported that the four Aloe spp. mainly differed in respect of number, size and distribution in DAPI-bands (AT-repeats). In the present investigation, different types of CMA-banding pattern accompanied by paracentric inversion and deletion to the banded region were noted. A variation in nature of satellites was found in their karyotypes. Therefore, GC-rich repeats together with inversion, deletion and the nature of satellites were involved in the karyotypic diversification of these species. Moreover, numerical aberration like segmental allopolyploidy and autopolyploidy may result in the formation of $H$. limifolia and $H$. reinwardtii, respectively.

\section{Acknowledgement}

We are grateful to Dr. M. Akhtaruzzaman, Professor of Botany, University of Dhaka, Bangladesh for constructive criticism and going through the manuscript. 


\section{References}

Alam, Sk. S. and Kondo, K. 1995. Differential staining with Orcein, Giemsa, CMA and DAPI for comparative chromosome study of 12 species of Australian Drosera (Droseraceae). American J. Bot. 82(10): 1278-1286.

—, - - and Hoshi, Y. 1995. Study on diffused centromeric nature of Drosera chromosomes. Cytologia 60: $43-47$.

— and Khanam, N. 2005. Fluorescent karyotype analysis of four Aloe species. Bangladesh J. Bot. 34: 17-20.

Bose, T. K., Chowdhury, B. and Sharma, S. P. 1991. Tropical Garden Plants (Reprint December 2001). Horticulture and Allied Publishers. 3B Madhab Chatterjee Street, Kolkata 700020, India.

Brandham, P. E. 1969. Chromosomal behaviour in the Aloineae: II. The frequency of interchange heterozygosity. Chromosoma 27: 216-225.

- 1971. The chromosomes of the Liliaceae II. Polyploidy and karyotype variation in the Aloineae. Kew Bulletin 25: 381399.

- 1973. The chromosomes of Liliaceae III. New cases of interchange hybridity in the Aloineae. Kew Bulletin 28: 341348.

- 1974. Interchange and inversion polymorphism among populations of Haworthia reinwardtii var. chalumnesis. Chromosoma 47: 85-108.

- 1975. Stabilized breakage of a duplicated chromosome segment in Aloe. Chromosoma 51: 269-278.

Brummitt, R. K. 1992. Vascular plant families and genera. Royal Botanic Gardens Kew, UK.

Darlington, C. D. and Kefallinou, M. 1957. Correlated chromosome aberrations at meiosis in Gasteria. Chromosoma 8: 364-370.

Hizume, M., Abe, K. K. and Tanaka, A. 1988. Fluorescent chromosome bandings in the Taxodiaceae. La Kromosomo 11-50: 1609-1619.

Kondo, T. and Hizume, M. 1982. Banding for the chromosomes of Cryptomeria japonica D. Don. Japan J. Forestry Soc. 64: $356-358$.

Riley, H. P. and Majumdar, S. K. 1979. The Aloineae, a biosystematic survey. Lexingtion, Kentucky. University Press of Kentucky.

Schweizer, D. 1976. Reverse fluorescent chromosome banding with Chromomycin and DAPI. Chromosoma 58: 307-324.

Sharma, A. K. and Chatterjii, A. K. 1958. Chromosome studies as a means of detecting the method of speciation in some members of Liliaceae. Genetica Iberica X: 179-178.

Stebbins, G. L. 1971. Chromosomal evolution in higher plants. Addison-Wesley Publishing Company, California, USA. pp. 208.

Taylor, W. R. 1925. Cytological studies on Gasteria II. A comparison of the chromosomes of Gasteria, Aloe and Haworthia. Amer, J. Bot. 12: 219-223.

Vladimir, S. 1963. A contribution to morphology of the nucleus of Aloe. The Nucleus 6(2): 181-184.

Vosa, C. G. and Bayer, M. B. 1986. Chromosome studies in the southern African flora. 38-57: Haworthia. Caryologia 39: 325-334. 